\title{
Mercury sample return to revolutionize our understanding of the solar system
}

\author{
A White Paper in support of the 2023-2032 Planetary Science and Astrobiology Decadal \\ Survey
}

Lead Author: Kathleen E. Vander Kaaden, Phone: 281-244-5020, Affiliation: Jacobs, JETS Contract, NASA Johnson Space Center (JSC), Kathleen.E.VanderKaaden@ nasa.gov

Co-Authors: Michelle S. Thompson (Purdue University), Francis M. McCubbin (NASA JSC), Catherine L. Johnson (University of British Columbia, Planetary Science Institute), Carolyn M. Ernst (Johns Hopkins University (JHU) Applied Physics Lab (APL)), Nancy L. Chabot (JHUAPL), Paul K. Byrne (North Carolina State University)

\section{Endorsers:}

Zoë Wilbur (University of Arizona), Arya Udry (University of Nevada Las Vegas), Jay Thompson (Jacobs/NASA JSC), Weijie Sun (University of Michigan Ann Arbor), Kevin Righter (NASA JSC), Soumya Ray (Arizona State University), Stephen Parman (Brown University), Maurizio Pajola (INAF-OAPD Astronomical Observatory of Padova), Prajkta Mane (Lunar and Planetary Institute (USRA)/NASA JSC), Alice Lucchetti (INAF-OAPD Astronomical Observatory of Padova), Christian Klimczak (University of Georgia), Peter James (Baylor University), Hope Ishii (University of Hawaii at Manoa), Munir Humayun (Florida State University), Steven Hauck, II (Case Western Reserve University), Lydia Hallis (University of Glasgow), Maria Gritsevich (Finnish Geospatial Research Institute), Marc Fries (NASA JSC), Stephen Elardo (University of Florida), Nicholas Dygert (University of Tennessee), Chuanfei Dong (Princeton University), Ariel Deutsch (Brown University), Jessica Barnes (University of Arizona), Rosalind Armytage (Jacobs/NASA JSC) 


\subsection{INTRODUCTION}

Our knowledge of the formation and evolution of the solar system is constantly changing with each new set of spacecraft data, meteorite analysis, and sample collected from another planetary body. Given the vast amount of data we have from Mars from orbiters [e.g., 1], landers [e.g., 2], rovers [e.g., 3], and meteorites [e.g., 4], increasing our understanding of the formation, makeup, and evolution of Mercury through similar efforts will help to constrain models of the lateral heterogeneities of the inner planetary disk and answer transformative solar system science questions (Table 1). Although we have greatly expanded our knowledge of Mercury with the return of data from the NASA MErcury Surface, Space ENvironment, GEochemistry, and Ranging (MESSENGER) mission [e.g., 5], there are still outstanding, fundamental questions that can only be answered via high-precision analyses conducted in Earth-based laboratories of materials directly sampled from the planet. Here we leverage information and ideas from Vander Kaaden et al. [6] to recommend sample return from Mercury be considered in future exploration efforts.

Table 1. Transformative Solar System Science Questions. Questions are not listed in order of priority. List is not exhaustive. Table modified from Vander Kaaden et al. [6].

\begin{tabular}{|l|c|}
\hline \multicolumn{1}{|c|}{ Science Question } & $\begin{array}{c}\text { High-precision Laboratory } \\
\text { Measurements }\end{array}$ \\
\hline $\begin{array}{l}\text { What can we learn about the composition of the Sun from } \\
\text { Mercury's regolith? }\end{array}$ & geochemical analyses \\
\hline $\begin{array}{l}\text { What can the composition of Mercury tell us about } \\
\text { exoplanet formation? }\end{array}$ & $\begin{array}{c}\text { geochemical, mineralogical, and } \\
\text { isotopic analyses }\end{array}$ \\
\hline $\begin{array}{l}\text { What does the present metal:silicate ratio of Mercury tell } \\
\text { us about the chemical composition across the inner Solar } \\
\text { System? }\end{array}$ & geochemical and isotopic analyses \\
\hline $\begin{array}{l}\text { What do the volatile abundances of Mercury tell us about } \\
\text { volatile distribution of the inner Solar System? }\end{array}$ & geochemical and isotopic analyses \\
\hline $\begin{array}{l}\text { Were there lateral heterogeneities in the protoplanetary } \\
\text { disk with distance from the Sun? }\end{array}$ & geochemical and isotopic analyses \\
\hline $\begin{array}{l}\text { Is Mercury enriched or depleted in nucleosynthetic } \\
\text { anomalies that are abundant in other Solar System objects? }\end{array}$ & isotopic analyses \\
\hline $\begin{array}{l}\text { What are the radiogenic ages of some of Mercury's surface } \\
\text { materials and what does this tell us about ongoing } \\
\text { processes in the inner Solar System? }\end{array}$ & age dating of crater material \\
\hline $\begin{array}{l}\text { What is the chronology of Mercury's geological evolution } \\
\text { and how does it compare to other bodies such as the Moon? }\end{array}$ & age dating of crater material \\
\hline $\begin{array}{l}\text { What are the microstructural, chemical, and optical } \\
\text { signatures of space weathering closer to the Sun? }\end{array}$ & coordinated analyses at the nano- \\
\hline
\end{tabular}

The first flybys of Mercury were conducted in 1974 and 1975 by the Mariner 10 spacecraft. This initial exploration of Mercury yielded images from $~ 45 \%$ of the planet, detection of a magnetic field, and measurements of elements such as $\mathrm{H}, \mathrm{He}$, and $\mathrm{O}$ in the exosphere, along with determining other physical characteristics of the planet [e.g., 7]. With this limited dataset, however, 
much information about Mercury still had to be inferred. Fortunately, NASA's MESSENGER mission provided the first in-depth study of the innermost planet.

Table 2. Transformative Mercury Science Questions. Questions are not listed in order of priority. List is not exhaustive. Table modified from Vander Kaaden et al. [6].

\begin{tabular}{|c|c|}
\hline Science Question & $\begin{array}{l}\text { High-precision Laboratory } \\
\text { Measurements } \\
\end{array}$ \\
\hline $\begin{array}{l}\text { What is the mineralogy of the largest volcanic plains unit } \\
\text { on the planet, Borealis Planitia? }\end{array}$ & mineralogical analyses \\
\hline $\begin{array}{l}\text { What is the composition of the low-reflectance material on } \\
\text { the planet, hypothesized to be the primary crust? }\end{array}$ & $\begin{array}{l}\text { geochemical and mineralogical } \\
\text { analyses }\end{array}$ \\
\hline What are the carriers of remanant magnetism on Mercury? & $\begin{array}{l}\text { geochemical, mineralogical and } \\
\text { magnetic properties analyses }\end{array}$ \\
\hline $\begin{array}{l}\text { How did Mercury differentiate to form its large core and } \\
\text { how did this process compare to the other terrestrial } \\
\text { planets? }\end{array}$ & $\begin{array}{l}\text { geochemical, mineralogical, } \\
\text { isotopic analyses }\end{array}$ \\
\hline $\begin{array}{l}\text { What are the composition constraints on Mercury's interior } \\
\text { units, such as its solid core, liquid core, mantle, and } \\
\text { potential other layers? }\end{array}$ & $\begin{array}{l}\text { geochemical, mineralogical, } \\
\text { isotopic analyses }\end{array}$ \\
\hline $\begin{array}{l}\text { What is the composition of the hollows, and how did they } \\
\text { form? }\end{array}$ & geochemical and isotopic analyses \\
\hline $\begin{array}{l}\text { Did Mercury undergo a giant impact early in its history or } \\
\text { did it accrete with the metal-silicate ratio it has? }\end{array}$ & geochemical and isotopic analyses \\
\hline Are the circum-Caloris plains volcanic or impact in origin? & geochemical and isotopic analyses \\
\hline $\begin{array}{l}\text { What is the age of major units on Mercury like Caloris } \\
\text { basin or the northern volcanic plains? }\end{array}$ & age dating and isotopic analyses \\
\hline $\begin{array}{l}\text { When did widespread effusive volcanism cease on } \\
\text { Mercury? }\end{array}$ & age dating and isotopic analyses \\
\hline $\begin{array}{l}\text { What are the microstructural, chemical, and optical } \\
\text { signatures of space weathering in samples from Mercury? }\end{array}$ & $\begin{array}{l}\text { coordinated analyses at the nano- } \\
\text { scale }\end{array}$ \\
\hline $\begin{array}{l}\text { How can the data from MESSENGER and eventually } \\
\text { BepiColombo be refined with new ground-truth data? }\end{array}$ & $\begin{array}{c}\text { geochemical, mineralogical, } \\
\text { isotopic analyses, age dating, } \\
\text { coordinated analyses at the nano- } \\
\text { scale }\end{array}$ \\
\hline
\end{tabular}

Orbiting Mercury from 2011 to 2015, cameras onboard the MESSENGER spacecraft were able to image the entirety of the planet and provide the first global view of Mercury. Coupling this imagery with data from other instruments onboard MESSENGER, we have gained a better understanding of the geochemical terranes associated with the surface materials on the planet and the unique nature of Mercury's geochemical state compared with the other terrestrial planets [e.g., 8]. MESSENGER also provided data that facilitated great advancements in our understanding of the geology, internal structure, exosphere, and magnetosphere of Mercury. These past mission 
datasets show that Mercury is a volatile-rich planet, has extensive polar deposits, exhibits a northsouth asymmetry in its global magnetic field, experienced prolonged global contraction, but a truncated history of volcanism, and has a dynamic magnetosphere as well as a seasonal exosphere. But the data from MESSENGER also revealed Mercury to be a geochemical end-member among the terrestrial planets, leaving many unanswered questions and prompting new ones that necessitate continued exploration of the planet well beyond current missions (Tables 1 and 2).

\subsection{FUTURE EXPLORATION OF MERCURY}

Following the general strategy of exploration of other planets, this continued exploration of Mercury should be conceived as a multi-mission, multi-generational effort (e.g., a sequence comprising flyby, orbiter, lander/rover, and sample return). Currently, there is only one ongoing mission to continue exploring the Sun's closest planetary neighbor. Launched on October 20, 2018, the joint ESA/JAXA dual-orbiter spacecraft BepiColombo is due to arrive at Mercury in December of 2025 and is the most ambitious effort yet attempted to explore Mercury [e.g., 9]. The Mercury Planetary Orbiter includes 11 scientific instruments and is set to study the surface, the internal composition of the planet, the magnetosphere, and the exosphere in unprecedented detail. The second spacecraft, "Mio" (formerly the Mercury Magnetospheric Orbiter), will characterize Mercury's dynamic magnetosphere and its interaction with the solar wind plasma and interplanetary magnetic field, as well as sodium emissions and dust monitoring. Although there are not any currently planned missions to Mercury beyond BepiColombo, the continued exploration of the planet has strong support within the scientific community [10]. There are several major knowledge gaps regarding Mercury's character and evolution where our current understanding could be dramatically improved with data acquired from the planet's surface via in situ landed science. Specifically, a Mercury lander would greatly advance our understanding of the planet's geochemical makeup, its interior structure, geological evolution, the present-day processes at work there, and even the planet's polar volatile inventory [10]. Although the data from remote-sensing missions provide a wealth of knowledge regarding the physical and chemical characteristics of a planetary body, and in situ analyses of the surface materials will transform our current understanding of Mercury, there are critical science questions that can best be addressed via examination of a sample in Earth-based laboratories, where numerous highly sensitive analytical measurements are possible (Tables 1 and 2). Here we detail the vast amount of information and transformative solar system science that can be achieved via sample return from Mercury. Crucially, a landed mission must be carried out prior to sample return to assess engineering constraints, determine appropriate sample site location(s), and facilitate the requirements for curation and handling of a Mercurian sample. As demonstrated by ongoing analysis of Apollo lunar samples, a returned sample from Mercury would be an invaluable scientific resource for generations to come, enabling the most sophisticated measurements to be brought to bear for decades and helping to further unlock the mysteries of our solar system.

\subsection{A NEW WINDOW INTO THE SOLAR SYSTEM}

The history of our solar system is recorded within the astromaterials that it comprises. At present, we have samples of Earth, the Moon, Mars, and numerous asteroids and comets. We do not have recognized samples from Mercury or Venus, nor of any of the outer solar system planets or satellites. The limited subset of materials we have to construct the history of our solar system results in many unanswered questions (Table 1). A sample from Mercury would enable 
comparative planetology studies, and would yield transformative science relevant to the origin, timing, and composition of planetary building blocks in the solar system.

\subsection{INSIGHTS INTO THE PROTOPLANETARY DISK}

There are four terrestrial planets (and associated moons) in the inner solar system along with numerous asteroids, thought to be relict planetesimals that survived the epoch of planet formation. These planetesimals provide the earliest history of our solar system [e.g., 11], forming within the first $0.5-8 \mathrm{Ma}$ after the formation of the first solids in the protoplanetary disk. Of the four terrestrial planets, Mercury and Mars are of a relatively small size such that they likely comprise only 1-2 planetary embryos [12]. In contrast, Earth and Venus likely consist of tens of planetary embryos [12]. If Mars and Mercury are relict individual planetary embryos or are composed of a small number of embryos, they can provide a crucial record of the period of solar system evolution between the formation of planetesimals and the growth of planets. Moreover, given the limited feeding zones of planetary embryos, samples of Mars and Mercury can provide unique insights into lateral heterogeneities within the inner portion of the protoplanetary disk. If these worlds' current positions are indicative of where they formed, samples from Mercury and Mars would yield information about the conditions in the innermost and outermost regions of the terrestrial planet-forming region of the protoplanetary disk. Aside from Mars, Mercury is the only remaining relict planetary embryo from which information about the planet-formation epoch of our solar system history can be attained. Samples from Mercury would therefore help to refine models of protoplanetary disk chemistry and the available building blocks of the terrestrial planets. Sample return from Mercury could provide important insights into the origin of nucleosynthetic isotope anomalies and early solar system chronology. Furthermore, the residual planetesimals that have been sampled (i.e., meteorites) may not represent all of the primary planet-building materials that were available at the time of planet formation, and samples from Mercury could lend insights into previously unsampled solar system building blocks.

\subsection{GEOCHEMICAL BEHAVIOR OF ELEMENTS AT REDUCED CONDITIONS}

The oxygen fugacity $\left(f \mathrm{O}_{2}\right)$, or partial pressure of $\mathrm{O}_{2}$ gas that is in equilibrium with a magmatic system, plays an important role in the physicochemical properties of that system, because $f \mathrm{O}_{2}$ has a substantial impact on the partitioning behavior and solubility of elements in various phases. The reducing nature of Mercury has raised many questions regarding the geochemical behavior of lithophile (oxygen-loving) elements in magmas at low oxygen fugacity (i.e., reduced conditions). Samples of Mercury would provide unparalleled insights into the distributions of major, minor, and trace elements among metal, sulfide, silicate, and oxide phases under these reduced conditions. Mercury returned samples would also fill a critical gap in our understanding of the behavior of elements under reducing conditions in complex geological systems. Furthermore, when combined with the geochemical behavior of elements under more oxidizing conditions, these data would help to constrain models of element distribution and behavior over a wide range of conditions relevant to exoplanetary systems. Finally, constraints on the elemental behavior exhibited by samples from Mercury are a prerequisite to using those samples to determine the bulk composition of the planet, including that of its core, mantle, and crust.

\subsection{THE STORAGE OF $\mathrm{H}_{2} \mathrm{O}$ IN PLANETARY INTERIORS}

Mercury has surface features indicating past explosive volcanism, consistent with the presence of magmatic volatiles in its interior [e.g., 15]. The surface of Mercury is also enriched in 
moderately volatile elements such as $\mathrm{K}, \mathrm{Na}, \mathrm{S}$, and $\mathrm{Cl}$. However, enrichments in moderately volatile elements are not typically a good indicator of the abundances of more volatile components, such as $\mathrm{H}_{2} \mathrm{O}$ [16]. In fact, the highly reducing conditions of Mercury's interior may limit the abundance of $\mathrm{H}_{2} \mathrm{O}$, given the effect of $f \mathrm{O}_{2}$ on the fugacity ratio of $\mathrm{H}_{2} \mathrm{O} / \mathrm{H}_{2}$ via the reaction $\mathrm{H}_{2}+$ $1 / 2 \mathrm{O}_{2} \leftarrow \rightarrow \mathrm{H}_{2} \mathrm{O}$ [e.g., 17]. If the estimated $\mathrm{fO}_{2}$ range of Mercury's mantle is an accurate predictor of the molecular $\mathrm{H}_{2} \mathrm{O} / \mathrm{H}_{2}$ ratio of Mercury's mantle, then the $\mathrm{H}_{2} \mathrm{O} / \mathrm{H}_{2}$ ratio is in the range of $0.034-$ 0.002 over a temperature range of 1300-1800 K [16]. Samples of Mercury would enable a test of this hypothesis, providing crucial insights into storage mechanisms of both $\mathrm{H}_{2}$ and $\mathrm{H}_{2} \mathrm{O}$ in the interiors of reduced planetary bodies. When coupled with knowledge of storage of $\mathrm{H}$ in more oxidized planetary bodies, $\mathrm{H}$ storage mechanisms in reduced planetary bodies would have important implications for the storage of $\mathrm{H}$ in exoplanets that may exhibit a range of $\mathrm{fO}_{2}$ similar to those for the terrestrial planets.

\subsection{SPACE WEATHERING ON AIRLESS BODIES}

Space weathering modifies the surfaces of airless bodies such as Mercury via micrometeorite impacts and solar wind irradiation. Together, these processes alter the microstructure, chemical composition, and reflectance properties of surface materials [e.g., 13]. The effects of space weathering complicate the interpretation of remotely sensed data and the subsequent characterization of planetary surfaces. Our understanding of space weathering across the inner solar system is based predominantly on the analysis of returned samples from the lunar surface and from asteroid Itokawa [e.g., 14]. However, the space weathering environment at Mercury is substantially different than the Moon and asteroid Itokawa in two important ways: 1) the composition of Mercury's surface is not fully characterized; and 2) the intensity of solar wind irradiation and the flux and velocity of micrometeorites impacting the surface is greater. With lower Fe but higher S contents expected on the surface of Mercury than on other airless bodies, returned sample analysis would enable the exploration of a new parameter space for the development of microstructural and chemical signatures of space weathering and, in turn, their influence on optical characteristics. Similarly, we have a very limited understanding of the role of $\mathrm{C}$ in the space weathering process, and so examining the effect of $\mathrm{C}$-bearing phases on the overall space weathering of airless surfaces, including Mercury, would be relevant for many carbonaceous surfaces across the solar system. Returning samples from Mercury would provide an end-member dataset by enabling the analysis of materials with unique starting compositions that have likely experienced extreme weathering rates, dominated by constituent processes unlike any other airless surface in the solar system.

\subsection{A NEW WINDOW INTO MERCURY'S FORMATION AND EVOLUTION}

In addition to providing insights into the makeup and evolution of the solar system, a sample from Mercury is also crucial to increasing our understanding of the geochemistry, mineralogy, geology, tectonism, and magnetism of the planet itself, and our efforts to begin to answer questions about its formation and evolution as a whole (Table 2).

\subsection{DIFFERENTIATION AND GEOCHEMICAL EVOLUTION}

Several instruments onboard MESSENGER, including a gamma ray spectrometer, a neutron spectrometer, and an X-ray spectrometer, collected geochemical data from the surface of Mercury, but these data are limited in terms of spatial extent, resolution, and the elements analyzed. 
Furthermore, MESSENGER did not carry instruments capable of examining the oxidation state of the measured elements, nor their host mineral(s), although BepiColombo will shed light on these properties. Such information is crucial to refining our understanding of the thermal and magmatic evolution of Mercury including bulk composition, crust formation, volcanism, smelting events, and the mineralogy and petrology of the planet [e.g., 18, 19]. Measurements of the mineralogy and siderophile element abundances in a sample from Mercury would enable modeling of the core formation process on this highly reduced planet, as has been done for Earth, Mars, and other planetary bodies. Such measurements would directly test the proposed hypotheses for Mercury's geochemical evolution and provide key new insight into the conditions and composition involved during Mercury's differentiation. In addition, better constraints on Mercury's bulk composition, including siderophile trace element data, would further enable comparative planetology studies, lending important insights into the planetary building blocks from which Mercury was assembled and its subsequent differentiation and expression of these materials on the surface.

\subsection{CHRONOLOGY AND GEOLOGIC EVOLUTION}

The geologic time scale of Mercury is segmented into 5 time-stratigraphic eras. These systems were originally defined as analogs to the lunar time-stratigraphic system, under the assumption that lunar impact flux models could be applied to Mercury [e.g., 20]. More recent analysis with MESSENGER data has led to revised age estimates for this system. Given these better-refined estimates from higher-resolution datasets, the shift in the positions of the time-stratigraphic boundaries has implications for the geologic history of Mercury, as these updated age constraints have major implications for models of the cooling of the planet and subsequent volcanic activity. However, these dates are based on models derived from crater statistics, rather than from measured radiometric age constraints, and are not supported by independent data from Mercury. Acquiring absolute age data would be instrumental in refining the geologic timescale for Mercury.

\subsection{TECTONISM AND GEOMECHANICAL PROPERTIES}

The history of tectonism on Mercury is dominated by global contraction - a process in which the planetary volume has decreased as the interior cooled through time. The results of this process manifest on Mercury as a planet-wide population of crustal shortening structures [21], most of which boast relief of a few hundred meters but the largest of which stand several kilometers high and are hundreds of kilometers long [22]. The acquisition of a sample from the surface of Mercury would provide valuable insight into the tectonic evolution and behavior of the planet. For example, the mineralogical properties of a sample would yield information regarding its mechanical characteristics, which play important roles in how tectonic deformation is manifested. Furthermore, the physical condition of a sample would yield useful information regarding the mechanical characteristics of Mercury's crust. Appraising the physical state of the sample in terms of fracture density, porosity, permeability, etc. would return invaluable insight into the bulk mechanical properties of the crust in general, with useful information that could be extended to the surfaces of other planetary bodies for which samples are not available.

\subsection{PAST AND ONGOING MAGNETISM}

The MESSENGER mission led to a wealth of discoveries regarding Mercury's magnetic field. In addition to characterizing the global dynamo field, and the surrounding magnetospheric environment, magnetic field data obtained at altitudes below $\sim 50 \mathrm{~km}$ toward the end of the orbital 
mission led to the discovery of weak, spatially localized signals from magnetized rocks [e.g., 23]. Although the discovery of crustal magnetization and the inferred geographical distribution over the northern hemisphere are robust, fundamental questions remain. First, the depth extent of magnetization and the iron mineralogy(ies) responsible for the magnetization are unknown. Second, remanent magnetization places tight constraints on the thermal evolution of the planet because it requires a dynamo field present at the time of acquisition of remanence [ 3.7 Ga; 23]. Sample return would enable the identification of the magnetic phases and laboratory experiments to establish their magnetic properties. Knowledge of these phases would enable direct comparisons with enstatite meteorites thought to contain magnetic mineralogies analogous to Mercury. Measurements of magnetic susceptibility (low-field and thermal) and the Curie temperature would enable accurate characterization of the magnetization induced in the present-day magnetic field. Furthermore, direct measurement of the remanent magnetization in a sample would constrain the strength of the ancient field, as has been possible from Apollo samples for the Moon.

\subsection{CONCLUSIONS AND RECOMMENDATIONS}

To ensure the continued exploration of the innermost planet, mission concepts to explore Mercury from both landed science and sample return need to be undertaken now. Because of the requirement to shed velocity on approach to Mercury from Earth, a mission dispatched to the planet takes several years to reach its destination, comparable to the cruise duration for an outer planets mission. Time is therefore of the essence. Lander science and sample return from Mercury would facilitate transformative solar system science that would place new and vital constraints on the building blocks and thermochemical evolution of Mercury and the terrestrial planets. Given the substantial investment of resources that would be needed to collect a sample from Mercury and bring it to Earth, it is critical that all challenges and risks are assessed and overcome prior to any sample return attempts, and that the material returned from the planet be diverse and curated appropriately [6]. Any delay in future exploration efforts could have detrimental impacts on the continued exploration of Mercury and, with it, our understanding of our solar system at large.

\subsection{REFERENCES}

1. Rogers, A.D. et al. JGR:Planets, 2015. 120(1): p. 62-91. 2. Arvidson, R.E., et al. Revs of Geophys, 1989. 27(1): p. 39-60. 3. Ming, D.W., et al. JGR: Planets, 2008. 113: p. E12. 4. McSween, H.Y. Meteoritics, 1994. 29(6): p. 757-779. 5. Solomon, S.C., et al. Cambridge Planetary Science, ed. F. Bagenal, et al. 2018, 583. 6. Vander Kaaden, K.E., et al. Space Sci Revs, 2019. 215(49). 7. Broadfoot, A.L., et al. GRL, 1976. 3: p. 577-580. 8. Weider, S.Z., et al. EPSL, 2015. 416: p. 109-120. 9. Benkhoff, J., et al. PSS, 2010. 58: p. 2-20. 10. Byrne, P.K., et al. https://bit.ly/2GqsmC2, 2018. 11. Wadhwa, M., et al. GCA, 2009. 73(17): p. 5189-5201. 12. Chambers, J.E. EPSL, 2004. 233: p. 241-252. 13. Domingue, D.L., et al. Space Sci Revs, 2014. 181: p. 121-214. 14. Thompson, M.S., et al. Earth, Planets, and Space, 2014. 66(1): p. 89. 15. Kerber, L., et al. EPSL, 2009. 285(3-4): p. 263-271. 16. McCubbin, F.M. et al. EPSL, 2019. 526: p. 115771. 17. Hirschmann, M.M., et al. EPSL, 2012. 345: p. 38-48. 18. Byrne, P.K., et al. GRL, 2016. 43: p. 7408-7416. 19. Vander Kaaden, K.E., et al. GCA, 2015. 149: p. 1-20. 20. Shoemaker, et al. in The Moon, 1962, Academic Press: New York. p. 289-300. 21. Strom, R.G et al. JGR, 1975. 80: p. 2478-2507. 22. Byrne, P.K., et al. in Mercury: The View after MESSENGER, 2019, Cambridge Univeristy Press. 23. Johnson, C.L., et al. in Mercury: The View after MESSENGER, 2019, Cambridge Univeristy Press. 